\section{DEVELOPMENT OF A GPS-GIS-BASED ROAD SIGNAGE MANAGEMENT SYSTEM FOR SELECTED ARTERIAL ROADS}

\author{
O. O. Adeleke ${ }^{a *}$, K. O. Akande ${ }^{a}$, S. S. Kolo ${ }^{b}$, J. O. Odumosu', O. O. \\ Sijuwola ${ }^{a}$ A. M. Ayanshola ${ }^{d}$
}

${ }^{a}$ Department of Civil Engineering, University of Ilorin, llorin, Nigeria

${ }^{b}$ Department of Civil Engineering, Federal University of Technology, Minna, Nigeria

'Department of Surveying and Geoinformatics, Federal University of Technology, Minna, Nigeria

${ }^{\mathrm{d} D e p a r t m e n t}$ of Water Resources and Environmental Engineering, University of llorin, llorin, Nigeria
Article history

Received

04 February 2020

Received in revised form

13 May 2020

Accepted

15 May 2020

Published online

31 July 2020

*Corresponding author adeleke.oo@unilorin.edu.ng

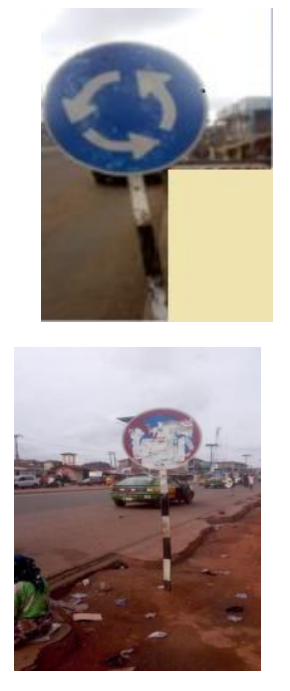

\begin{abstract}
Traffic signs, pavement markings and other road safety assets such as speed bumps are provided on roadway facilities to enhance traffic flow and safety. They will however perform their functions effectively if they meet the basic requirements of i. fulfill a need ii. command attention iii. convey a clear simple meaning iv. command respect from road users and v. give adequate time for proper response. It is noticed and rightfully observed by the Nigeria National Council on Works that these assets on Nigeria roads are not adequately managed and maintained. Some of them are not well positioned, some are missing and some others are damaged. There is therefore the need to assess the existing roadway assets, their conditions and adequacy, and generate a database needed for their effective operation, management and maintenance. The study thus aims at addressing the observed inadequacies by proffering a GPS-GIS-based solution. The objectives are to i. carry out an inventory of existing road signs and markings; ii. determine their adequacy or otherwise and iii. develop a GIS- based road signage management system. It is anticipated that the application of the developed system will enhance the performance of the agencies responsible for the operations of the road assets.
\end{abstract}

Keywords: GIS, safety, signage management, traffic flow, traffic sign defects

CC 2020 Penerbit UTM Press. All rights reserved

\section{$1.0 \quad$ INTRODUCTION}

\subsection{Background to the Study}

Traffic road signs, pavement markings and other road safety assets such as speed bumps are very useful on fixed facility as they display to road users what to do, what to watch for and where to drive; resulting to smoother traffic flow, reduced fuel consumption, improved roadway capacity and reduced collision rate (Federal Ministry of Works, 2013; McGee, 2010; PourRouholamin and Zhou 2016). The functionality of road signs and markings however, depends on their visibility and recognition during day time and night time. This calls for their adequate placement, management and maintenance (Yannis et. al., 2014). However, the sheer size of these assets can be enormous which poses a great challenge and demand on agencies saddled with their operations, which sometimes incapacitates their adequate maintenance. In Ilorin metropolis of Nigeria as it is also the case in some other urban centres in Nigeria, these road assets are noticed not to be adequately performing their basic functions of warning, guiding and providing information to road users as needful because some of them are defaced while others are damaged and need replacements (Nigerian National Council on Works (NNCW), 2017). Coupled with these are observed faded pavement markings on the city road network. Also noticeable is inadequate placement of some of these road assets. These 
deficiencies result to increased hazardous environment and travel risk for road users which sometimes lead to accidents. Accurate data on the locations, attributes and conditions of the traffic assets are important as they serve as the basis for cost efficient and compliance effective maintenance and management strategies (Migletz et. al., 1994; Boggs et. al., 2013). In the bid to improve on the operations, management and maintenance of these assets which require adequate and well managed data on their locations, attributes and conditions, the combination of Global Positioning System (GPS) and Geographic Information System (GIS) technologies are utilised in the study to develop a GPS-GIS-based management system. GPS is a satellite based navigation system that uses signals from satellites to determine the position (latitude and longitude) of a receiver at a location and also provides time information. The GPS provides the location and time information in any weather conditions, in any part of the world, for the entire day. Its area of application includes mapping and navigation (Bhawani, 2014). A GIS is a system of hardware, software and procedures used to facilitate the management, manipulation, analysis, modelling, representation and display of georeferenced or spatial data to solve complex problems regarding planning and management of resources. GIS has emerged as an essential tool for urban and resource planning and facility management among many other applications. Functions of GIS include: data entry, spatial query, data analysis and display, data management and information retrieval (Escobar et. al., 1990; Goodchild and Kemp, 1990). The study aims at addressing the inadequacies associated with the location and maintenance of road signage with a view to proffering a GPS-GIS-based solution to ameliorate the problem The objectives are to i. carry out an inventory of existing road signs and markings; ii. determine the adequacy or otherwise of existing road signs and markings and iii. develop a GPS-GISbased road signs management system. This paper presents a pilot study conducted on four roadways in llorin metropolis. The study can however be replicated on wider geographic area.

\subsection{Study Area}

Four primary arterial roads in Ilorin metropolis, Kwara State, Nigeria are selected for the study. The roads are Ibrahim Taiwo road, Unity road, Murtala Mohammed road and University of llorin Main Campus road.

\subsection{Literature Review}

Traffic signs can be grouped into several types. For example, Annex 1 of the Vienna Convention on Road Signs and Signals (United Nations, 1968), which on 30 June 2004 had 52 signatory countries, defines eight categories of signs: i. Danger warning signs ii. Priority signs iii. Prohibitory or restrictive signs iv. Mandatory signs v. Special regulation signs vi. Information, facilities, or service signs vii. Direction, position, or indication signs and viii. Additional panels. Traffic signs are also however functionally classified into three broad groups as i. regulatory signs ii. warning signs and iii. guide signs (Alberta Infrastructure and Transportation, 2006). Accurate understanding is a dominant factor for the effectiveness of a traffic sign system. With poor comprehension, memory for sign meanings and the likelihood of prompting effective driver reactions will decrease (Charlton, 2006). Lack of comprehension or misunderstanding of the message from these signs can cause negative consequences which may contribute to operational and safety problems (Government of Alberta, 2005; Ogden, Womack and Mounce, 1990; Ng and Chan, 2008). In order for traffic signs to be effective, they should meet the following basic requirements: 1 . Fulfill a need, 2. Command attention, 3 . Convey a clear, simple meaning, 4. Command respect from road users and 5 . Give adequate time for proper response (Federal Highway Administration, 2009). Road surface marking is any kind of device or material that is used on a road surface in order to convey official information. They are used on paved roadways to provide guidance and information to drivers and pedestrians (Texas Dept. of Transp., 2004; Pour-Rouholamin and Zhou, 2016). Uniformity of the markings is an important factor in minimizing confusion and uncertainty about their meaning, and efforts exist to standardize such markings across borders (United Nations, 1968). However, countries and areas categorize and specify road surface markings in different ways. Traffic marking performance can be evaluated under factors such as; general daylight appearance, color, film condition, bead retention, and reflectance. Several materials can be used for pavement markings. However, the performance and cost for each material may vary greatly depending on a number of factors, such as traffic volume, surface type, climatic condition and project duration (Praprut and Adam, 2011; Ozelim and Turochy, 2014). The most popularly used pavement marking materials are paint, thermoplastics, epoxy and polyuria (Texas Dept. of Transp., 2004; Rahman and Duggal, 2015). The popularly used material for pavement marking in Nigeria is thermoplastic paint (Punch, 2019). The performances of these marking materials are measured in terms of their durability, retro-reflectivity, and skid resistance. Durability refers to the amount of material remaining on the pavement surface over time and it affects both the day time and night time appearance of marking. In an effort to effectively manage traffic signs, some agencies and municipalities have developed GIS-based management systems of traffic signs examples include Kenya (Kiema and Mwangi, 2009) and Uttarakhand (Pandeh and Joshi, 2011).

\subsection{MATERIALS AND METHODS}

\subsection{Data Collection}

Primary and secondary data were used in building the signage inventory.

\subsubsection{Primary Data}

\subsubsection{Non Spatial Data}

The non spatial attribute and condition data used in the database were obtained through a visit to the study area and direct observation of the traffic signs, pavement markings and safety assets with the aid of camera and video coverage to capture them. Visual day time and night time physical condition inspections of the signs, pavement markings and safety assets were carried out manually to identify any deficiencies which impact visibility and legibility thus determining their functionality. The sign type, material used for the sign, the height, shape, color, positioning, photographs and adequacy of 
the traffic safety facilities were obtained and assessed. Tape was used to measure the heights as appropriate.

\subsubsection{Spatial Data}

The GPS was used to capture the coordinates of the locations of all the exiting traffic signs in the study area.

\subsubsection{Sign Condition Assessment}

The sign condition assessment data was used to rate and classify the traffic signs into: CRITICAL - sign needs immediate replacement or repair to restore performance to the desired level; FAIR - sign that may need replacement within six months to five years and GOOD - sign that may need replacement in five or more years. The date and time of inspections and inspectors' names, weather conditions were also captured for future reference.

\subsubsection{Secondary Data}

The secondary data used for this project was an existing road map of the study area which was digitized and georeferenced.

\subsection{Importation of the Collected Data into GIS Software and Data Analysis}

The data collected was used to build the sign inventory database. A folder was created for the collected attribute data of the features; the data was prepared in Microsoft Excel and imported into the ArcGIS10.3.1 through the tools on the interface. The data was then converted into shape file and added to the georeferenced line features (road network) through the Add data (+) on the interface to form the database.
The ArcGIS was thereafter used among other things to map out the critical, fair and good traffic signs. Arc Catalog was used to store the point data and their corresponding attributes. The Facility feature class is broken down into separate feature classes for the purpose of analysis.

\subsection{RESULTS AND DISCUSSION}

\subsection{Road Inventory}

\subsubsection{Unity Road}

Unity Road is a dual carriage road which is overlaid with flexible bituminous surfacing material. The road is $1.02 \mathrm{~km}$ long. The median width ranges between 0.4 to $0.6 \mathrm{~m}$. Lined drain runs along the edges of this fixed facility with width ranging from 0.6 to $0.9 \mathrm{~m}$. The major intersection along this road is Obbo road junction, Coca-Cola road junction and Irewolede junction. There is also a market adjacent to the road at Obbo road junction. All vehicle types are used on this road. Nevertheless, the road does not have pavement markings; neither lane markings, pedestrian crossings nor stoplines at intersections where appropriate. There are eleven traffic signs along the road. The traffic signs are mounted at a height of $2.5 \mathrm{~m}$ from ground elevation which is in accordance to Federal Ministry of Works (2013) specification. The sign inventory is shown in Table 1 . The table shows the coordinates, the post condition, the sign defect, the material used for the traffic signs, the information portrayed by each traffic sign and the shape of each traffic sign. This helps the traffic signs management personnel to have required and adequate details on each traffic sign. This is similarly done for the other studied roads as shown in Sections 3.1.2 - 3.1.4

Table 1 Data obtained on Unity Road

\begin{tabular}{|c|c|c|c|c|c|c|c|c|c|c|}
\hline $\begin{array}{l}\text { Sign/Post ID } \\
\text { Number }\end{array}$ & $\begin{array}{l}\text { Sign Street } \\
\text { Name }\end{array}$ & $\begin{array}{l}\text { Sign } \\
\text { Position }\end{array}$ & Latitude & Longitude & $\begin{array}{l}\text { Post } \\
\text { Material }\end{array}$ & $\begin{array}{l}\text { Post } \\
\text { Type }\end{array}$ & Sign Category & $\begin{array}{l}\text { Mou } \\
\text { nt } \\
\text { Heig } \\
\text { ht } \\
\text { (m) }\end{array}$ & Sign Defect & $\begin{array}{l}\text { Sign/P } \\
\text { ost } \\
\text { Condit } \\
\text { ion }\end{array}$ \\
\hline 1 & Unity Road & Right & $8^{\circ} 28^{\prime} 50.30^{\prime \prime} \mathrm{N}$ & $4^{\circ} 33^{\prime} 44.84^{\prime \prime E}$ & Steel & $\begin{array}{l}\text { Railway } \\
\text { Post }\end{array}$ & Information & 2.7 & NIL & Good \\
\hline 2 & Unity Road & Right & $8^{\circ} 28^{\prime} 50.18^{\prime \prime} \mathrm{N}$ & $4^{\circ} 33^{\prime} 44.92^{\prime \prime} \mathrm{E}$ & Steel & Round & Information & 2.5 & NIL & Good \\
\hline 3 & Unity Road & Center & $8^{\circ} 28^{\prime} 49.91^{\prime \prime} \mathrm{N}$ & $4^{\circ} 33^{\prime} 44.44^{\prime \prime} \mathrm{E}$ & Steel & Round & Information & 2.6 & NIL & Good \\
\hline 4 & Unity Road & Center & $8^{\circ} 28^{\prime} 49.05^{\prime \prime} \mathrm{N}$ & $4^{\circ} 33^{\prime} 44.92^{\prime \prime} \mathrm{E}$ & Steel & Round & Information & 2.5 & NIL & Good \\
\hline 5 & Unity Road & Left & $8^{\circ} 28^{\prime} 48.78^{\prime \prime} \mathrm{N}$ & $4^{\circ} 33^{\prime} 44.40^{\prime \prime} \mathrm{E}$ & Steel & Round & Information & 2.5 & NIL & Good \\
\hline 6 & Unity Road & Left & $8^{\circ} 28^{\prime} 49.09^{\prime \prime} \mathrm{N}$ & $4^{\circ} 33^{\prime} 43.65^{\prime \prime} \mathrm{E}$ & Steel & Square & Regulatory & 2.5 & NIL & Good \\
\hline 7 & Unity Road & Centre & $8^{\circ} 28^{\prime} 49.87^{\prime \prime} \mathrm{N}$ & $4^{\circ} 33^{\prime} 40.07^{\prime \prime} \mathrm{E}$ & Steel & Round & Information & 2.5 & NIL & Good \\
\hline 8 & Unity Road & Left & $8^{\circ} 28^{\prime} 49.60^{\prime \prime} \mathrm{N}$ & $4^{\circ} 33^{\prime} 39.84 " \mathrm{E}$ & Steel & Round & Regulatory & 2.5 & NIL & Good \\
\hline 9 & Unity Road & Right & $8^{\circ} 28^{\prime} 50.03^{\prime \prime} \mathrm{N}$ & $4^{\circ} 33^{\prime} 39.95 " \mathrm{E}$ & Steel & Round & Warning & 2.5 & Low visibility & Fair \\
\hline 10 & Unity Road & Right & $8^{\circ} 28^{\prime} 50.10^{\prime \prime} \mathrm{N}$ & $4^{\circ} 33^{\prime} 38.97 " \mathrm{E}$ & Steel & Round & Information & 2.5 & Low visibility & Fair \\
\hline 11 & Unity Road & Left & $8^{\circ} 28^{\prime} 49.68^{\prime \prime} \mathrm{N}$ & $4^{\circ} 33^{\prime} 39.17^{\prime \prime E}$ & Steel & Round & Information & 2.5 & NIL & Good \\
\hline
\end{tabular}

\subsubsection{Ibrahim Taiwo Road}

Ibrahim Taiwo Road is a $3.17 \mathrm{~km}$ long dual carriageway overlaid by a flexible bituminous surfacing material with double lanes in each direction. The median width ranges between 0.4 to $0.6 \mathrm{~m}$ with street-light within. Lined drains run along the edges of this fixed facility with width ranging from 0.6 to $0.9 \mathrm{~m}$. All vehicle types are used on this road. The major intersections along the road include Oro road junction and Oko-erin junction. The road does not have pavement markings. The road has five traffic 
signs. The traffic signs are mounted at a height of $2.0 \mathrm{~m}$ from ground elevation, which is within the range of traffic sign height specified by Federal Ministry of Works (2013). The sign inventory is presented in Table 2 .

\subsubsection{Murtala Mohammed Road}

Murtala Mohammed road has a total length of $10 \mathrm{~km}$. The double lane dual carriageway has shoulders along its sides with bituminous material overlaid on both the shoulders and the carriageway. The drainage system along the edges is covered by slabs to serve as sidewalk for pedestrians. Along the shoulders are road curbs to segregate the carriageway from the sidewalk. The shoulders have width range of 1.5 to $1.6 \mathrm{~m}$. The median width ranges from $0.5 \mathrm{~m}$ to $1.0 \mathrm{~m}$ with rails along the median to control road crossing by pedestrians. Major junctions along this road are: Challenge, Post-Office, UBA and Anjorin junctions etc. Challenge intersection is located within $200 \mathrm{~m}$ to $300 \mathrm{~m}$ from UBA junction. $300 \mathrm{~m}$ from the Challenge intersection towards Maraba intersection is a fly-over that spans the Post-office intersection. The fly-over has guardrails along the edges for vehicles on it, also white solid edge lines are drawn to inform users of both side restriction and lane crossing; the lane markings on the flyover are observed to have faded. The road has a total number of thirteen traffic signs, with no trace of pavement markings or stoplines at any of its intersections. The traffic signs are mounted at a height of $2.5 \mathrm{~m}$ from ground elevation which is in accordance to the Federal Ministry of Works (2013) specification. The sign inventory is presented in Table 3.

Table 2 Data Obtained on Ibrahim Taiwo Road

\begin{tabular}{|c|c|c|c|c|c|c|c|c|c|c|}
\hline $\begin{array}{l}\text { Sign/Post } \\
\text { ID } \\
\text { Number }\end{array}$ & $\begin{array}{l}\text { Sign Street } \\
\text { Name }\end{array}$ & $\begin{array}{l}\text { Sign } \\
\text { Position }\end{array}$ & Latitude & Longitude & $\begin{array}{l}\text { Post } \\
\text { Material }\end{array}$ & $\begin{array}{l}\text { Post } \\
\text { Type }\end{array}$ & Sign Category & $\begin{array}{l}\text { Mount } \\
\text { Height } \\
(\mathrm{m})\end{array}$ & $\begin{array}{l}\text { Sign } \\
\text { Defect }\end{array}$ & $\begin{array}{l}\text { Sign/Post } \\
\text { Condition }\end{array}$ \\
\hline 1 & $\begin{array}{l}\text { Ibrahim } \\
\text { Taiwo Road }\end{array}$ & Left & $8^{\circ} 28^{\prime} 57.44^{\prime \prime} \mathrm{N}$ & $4^{\circ} 33^{\prime} 14.38^{\prime \prime E}$ & Steel & $\begin{array}{l}\text { Not } \\
\text { shown }\end{array}$ & Not shown & 2.0 & $\begin{array}{l}\text { Mounted } \\
\text { head } \\
\text { removed }\end{array}$ & Critical \\
\hline 2 & $\begin{array}{l}\text { Ibrahim } \\
\text { Taiwo Road }\end{array}$ & Left & $8^{\circ} 28^{\prime} 57.40^{\prime \prime} \mathrm{N}$ & $4^{\circ} 33^{\prime} 11.74 " \mathrm{E}$ & Steel & $\begin{array}{l}\text { Not } \\
\text { shown }\end{array}$ & Not shown & 2.0 & $\begin{array}{l}\text { Mounted } \\
\text { head } \\
\text { removed }\end{array}$ & Critical \\
\hline 3 & $\begin{array}{l}\text { Ibrahim } \\
\text { Taiwo Road }\end{array}$ & Right & $8^{\circ} 28^{\prime} 57.79^{\prime \prime} \mathrm{N}$ & $4^{\circ} 33^{\prime} 11.86^{\prime \prime E}$ & Steel & Round & Information & 2.0 & Not visible & Critical \\
\hline 4 & $\begin{array}{l}\text { Ibrahim } \\
\text { Taiwo } \\
\text { Road }\end{array}$ & Left & $8^{\circ} 28^{\prime} 55.37^{\prime \prime N}$ & $4^{\circ} 32^{\prime} 47.49^{\prime \prime} \mathrm{E}$ & Steel & Round & Information & 2.0 & Not visible & Critical \\
\hline 5 & $\begin{array}{l}\text { Ibrahim } \\
\text { Taiwo Road }\end{array}$ & Left & $8^{\circ} 28^{\prime} 54.05^{\prime \prime N}$ & $4^{\circ} 32 ' 39.73^{\prime \prime E}$ & Steel & Round & Warning & 2.0 & Not visible & Critical \\
\hline
\end{tabular}

Table 3 Data Obtained on Murtala Mohammed Road

\begin{tabular}{|c|c|c|c|c|c|c|c|c|c|c|}
\hline $\begin{array}{l}\text { Sign/Post ID } \\
\text { Number }\end{array}$ & $\begin{array}{l}\text { Sign } \\
\text { Street } \\
\text { Name }\end{array}$ & $\begin{array}{l}\text { Sign } \\
\text { Position }\end{array}$ & Latitude & Longitude & $\begin{array}{l}\text { Post } \\
\text { Material }\end{array}$ & Post Type & $\begin{array}{l}\text { Sign } \\
\text { Category }\end{array}$ & $\begin{array}{l}\text { Mount } \\
\text { Height (m) }\end{array}$ & $\begin{array}{l}\text { Sign } \\
\text { Defect }\end{array}$ & $\begin{array}{l}\text { Sign/Post } \\
\text { Condition }\end{array}$ \\
\hline 1 & $\begin{array}{l}\text { Murtala } \\
\text { Mohd.Rd }\end{array}$ & Centre & $8^{\circ} 29^{\prime} 57.49^{\prime \prime} \mathrm{N}$ & $4^{\circ} 34^{\prime} 25.71 " \mathrm{E}$ & Steel & Round & Information & 2.5 & NIL & Good \\
\hline 2 & $\begin{array}{l}\text { Murtala } \\
\text { Mohd.Rd }\end{array}$ & Left & $8^{\circ} 29^{\prime} 58.97 " \mathrm{~N}$ & $4^{\circ} 34^{\prime} 27.95^{\prime \prime E}$ & Steel & Round & Information & 2.5 & $\begin{array}{l}\text { Not } \\
\text { visible }\end{array}$ & Critical \\
\hline 3 & $\begin{array}{l}\text { Murtala } \\
\text { Mohd.Rd }\end{array}$ & Right & $8^{\circ} 29^{\prime} 55.97 " \mathrm{~N}$ & $4^{\circ} 34^{\prime} 23.50^{\prime \prime E}$ & Steel & Round & Warning & 2.5 & $\begin{array}{l}\text { Low } \\
\text { visibility }\end{array}$ & Fair \\
\hline 4 & $\begin{array}{l}\text { Murtala } \\
\text { Mohd.Rd }\end{array}$ & Centre & $8^{\circ} 29^{\prime} 54.34^{\prime \prime} \mathrm{N}$ & $4^{\circ} 34^{\prime} 22.32 " \mathrm{E}$ & Steel & Square & Information & 2.5 & NIL & Good \\
\hline 5 & $\begin{array}{l}\text { Murtala } \\
\text { Mohd.Rd }\end{array}$ & Left & $8^{\circ} 29^{\prime} 49.97^{\prime \prime} \mathrm{N}$ & $4^{\circ} 34^{\prime} 18.78 " \mathrm{E}$ & Steel & Square & Information & 2.6 & NIL & Good \\
\hline 6 & $\begin{array}{l}\text { Murtala } \\
\text { Mohd.Rd }\end{array}$ & Left & $8^{\circ} 29^{\prime} 46.59^{\prime \prime} \mathrm{N}$ & $4^{\circ} 34^{\prime} 15.98^{\prime \prime E}$ & Steel & Round & Information & 2.5 & $\begin{array}{l}\text { Not } \\
\text { visible }\end{array}$ & Critical \\
\hline 7 & $\begin{array}{l}\text { Murtala } \\
\text { Mohd.Rd }\end{array}$ & Right & $8^{\circ} 29^{\prime} 36.38^{\prime \prime} \mathrm{N}$ & $4^{\circ} 34^{\prime} 06.49^{\prime \prime E}$ & Steel & Round & Information & 2.5 & $\begin{array}{l}\text { Not } \\
\text { visible }\end{array}$ & Critical \\
\hline 8 & $\begin{array}{l}\text { Murtala } \\
\text { Mohd.Rd }\end{array}$ & Left & $8^{\circ} 29^{\prime} 34.28^{\prime \prime} \mathrm{N}$ & $4^{\circ} 34^{\prime} 05.11 " \mathrm{E}$ & Steel & Round & Information & 2.3 & $\begin{array}{l}\text { Not } \\
\text { visible }\end{array}$ & Critical \\
\hline 9 & $\begin{array}{l}\text { Murtala } \\
\text { Mohd.Rd }\end{array}$ & Left & $8^{\circ} 29^{\prime} 30.81^{\prime \prime} \mathrm{N}$ & $4^{\circ} 34^{\prime} 01.37 " E$ & Steel & Round & Information & 2.5 & NIL & Good \\
\hline 10 & $\begin{array}{l}\text { Murtala } \\
\text { Mohd.Rd }\end{array}$ & Centre & $8^{\circ} 29^{\prime} 26.88^{\prime \prime} \mathrm{N}$ & $4^{\circ} 33^{\prime} 56.76 " \mathrm{E}$ & Steel & Square & Information & 2.5 & NIL & Good \\
\hline 11 & $\begin{array}{l}\text { Murtala } \\
\text { Mohd.Rd }\end{array}$ & Centre & $8^{\circ} 29^{\prime} 25.59^{\prime \prime} \mathrm{N}$ & $4^{\circ} 33^{\prime} 55.74 " \mathrm{E}$ & Steel & Square & Regulatory & 2.5 & $\begin{array}{l}\text { Low } \\
\text { visibility }\end{array}$ & Fair \\
\hline 12 & $\begin{array}{l}\text { Murtala } \\
\text { Mohd.Rd }\end{array}$ & Centre & $8^{\circ} 29^{\prime} 04.60^{\prime \prime} \mathrm{N}$ & $4^{\circ} 33^{\prime} 48.77^{\prime \prime E}$ & Steel & Square & Regulatory & 2.5 & NIL & Good \\
\hline 13 & $\begin{array}{l}\text { Murtala } \\
\text { Mohd.Rd }\end{array}$ & Centre & $8^{\circ} 29^{\prime} 01.33^{\prime \prime} \mathrm{N}$ & $4^{\circ} 33^{\prime} 48.22^{\prime \prime} \mathrm{E}$ & Steel & Round & Information & 2.5 & NIL & Good \\
\hline
\end{tabular}




\subsubsection{University of Ilorin Main Campus Road}

The road runs from University of Ilorin main gate to University of Ilorin roundabout (where there is a fountain). It is a $5.36 \mathrm{~km}$ 3-lane carriageway of a width of $9.10 \mathrm{~m}$. The road has twenty traffic signs. The traffic signs are mounted at a height of $2.1 \mathrm{~m}$ from ground elevation. It is overlaid by a flexible bituminous surfacing material. The road has fourteen bumps for speed calming. Bump information signs are however not provided for all the bumps nor are they marked with paints. There are also neither lane markings nor stoplines on the entire road length. The sign inventory is presented in Table 4.

Table 4 Data Obtained on University of Ilorin Road

\begin{tabular}{|c|c|c|c|c|c|c|c|c|c|c|}
\hline $\begin{array}{l}\text { Sign/Post } \\
\text { ID } \\
\text { Number }\end{array}$ & $\begin{array}{l}\text { Sign } \\
\text { Street } \\
\text { Name }\end{array}$ & $\begin{array}{l}\text { Sign } \\
\text { Position }\end{array}$ & Latitude & Longitude & $\begin{array}{l}\text { Post } \\
\text { Materi } \\
\text { al }\end{array}$ & Post Type & $\begin{array}{l}\text { Sign } \\
\text { Category }\end{array}$ & $\begin{array}{l}\text { Mount } \\
\text { Height } \\
\text { (m) }\end{array}$ & $\begin{array}{l}\text { Sign } \\
\text { Defect }\end{array}$ & $\begin{array}{l}\text { Sign/Post } \\
\text { Condition }\end{array}$ \\
\hline 1 & $\begin{array}{l}\text { Unilorin } \\
\text { Road }\end{array}$ & Left & $8^{\circ} 28^{\prime} 51.21^{\prime \prime} \mathrm{N}$ & $4^{\circ} 38^{\prime} 15.45^{\prime \prime E}$ & Steel & Triangle & Warning & 2.0 & NIL & Good \\
\hline 2 & $\begin{array}{l}\text { Unilorin } \\
\text { Road }\end{array}$ & Left & $8^{\circ} 28^{\prime} 51.32^{\prime \prime N}$ & $4^{\circ} 38^{\prime} 29.34^{\prime \prime E}$ & Steel & Round & $\begin{array}{l}\text { Informati } \\
\text { on }\end{array}$ & 2.1 & $\begin{array}{l}\text { Not } \\
\text { visible }\end{array}$ & Critical \\
\hline 3 & $\begin{array}{l}\text { Unilorin } \\
\text { Road }\end{array}$ & Right & $8^{\circ} 28^{\prime} 51.00^{\prime \prime N}$ & $4^{\circ} 38^{\prime} 34.54^{\prime \prime E}$ & Steel & Round & $\begin{array}{l}\text { Informati } \\
\text { on }\end{array}$ & 2.1 & $\begin{array}{l}\text { Not } \\
\text { visible }\end{array}$ & Critical \\
\hline 4 & $\begin{array}{l}\text { Unilorin } \\
\text { Road }\end{array}$ & Right & $8^{\circ} 28^{\prime} 50.20^{\prime \prime} \mathrm{N}$ & $4^{\circ} 38^{\prime} 39.71 " \mathrm{E}$ & Steel & Round & $\begin{array}{l}\text { Informati } \\
\text { on }\end{array}$ & 2.1 & NIL & Good \\
\hline 5 & $\begin{array}{l}\text { Unilorin } \\
\text { Road }\end{array}$ & Right & $8^{\circ} 28^{\prime} 44.69^{\prime \prime} \mathrm{N}$ & $4^{\circ} 38^{\prime} 48.10^{\prime \prime E}$ & Steel & Round & $\begin{array}{l}\text { Informati } \\
\text { on }\end{array}$ & 2.1 & $\begin{array}{l}\text { Not } \\
\text { visible }\end{array}$ & Critical \\
\hline 6 & $\begin{array}{l}\text { Unilorin } \\
\text { Road }\end{array}$ & Right & $8^{\circ} 28^{\prime} 34.33^{\prime \prime N}$ & $4^{\circ} 38^{\prime} 52.24^{\prime \prime E}$ & Steel & Round & $\begin{array}{l}\text { Informati } \\
\text { on }\end{array}$ & 2.1 & $\begin{array}{l}\text { Not } \\
\text { visible }\end{array}$ & Critical \\
\hline 7 & $\begin{array}{l}\text { Unilorin } \\
\text { Road }\end{array}$ & Right & $8^{\circ} 28^{\prime} 27.62^{\prime \prime N}$ & $4^{\circ} 38^{\prime} 53.34^{\prime \prime E}$ & Steel & Round & $\begin{array}{l}\text { Informati } \\
\text { on }\end{array}$ & 2.1 & $\begin{array}{l}\text { Not } \\
\text { visible }\end{array}$ & Critical \\
\hline 8 & $\begin{array}{l}\text { Unilorin } \\
\text { Road }\end{array}$ & Right & $8^{\circ} 28^{\prime} 20.12^{\prime \prime N}$ & $4^{\circ} 38^{\prime} 55.03^{\prime \prime E}$ & Steel & Round & $\begin{array}{l}\text { Informati } \\
\text { on }\end{array}$ & 2.1 & $\begin{array}{l}\text { Not } \\
\text { visible }\end{array}$ & Critical \\
\hline 9 & $\begin{array}{l}\text { Unilorin } \\
\text { Road }\end{array}$ & Right & $8^{\circ} 28^{\prime} 17.08^{\prime \prime} \mathrm{N}$ & $4^{\circ} 38^{\prime} 56.86^{\prime \prime} \mathrm{E}$ & Steel & Round & $\begin{array}{l}\text { Informati } \\
\text { on }\end{array}$ & 2.1 & $\begin{array}{l}\text { Not } \\
\text { visible }\end{array}$ & Critical \\
\hline 10 & $\begin{array}{l}\text { Unilorin } \\
\text { Road }\end{array}$ & Left & $8^{\circ} 28^{\prime} 12.22^{\prime \prime} \mathrm{N}$ & $4^{\circ} 39^{\prime} 02.94^{\prime \prime} \mathrm{E}$ & Steel & Round & $\begin{array}{l}\text { Informati } \\
\text { on }\end{array}$ & 2.1 & $\begin{array}{l}\text { Not } \\
\text { visible }\end{array}$ & Critical \\
\hline 11 & $\begin{array}{l}\text { Unilorin } \\
\text { Road }\end{array}$ & Right & $8^{\circ} 28^{\prime} 09.79^{\prime \prime N}$ & $4^{\circ} 39^{\prime} 06.86^{\prime \prime} \mathrm{E}$ & Steel & Round & $\begin{array}{l}\text { Informati } \\
\text { on }\end{array}$ & 2.1 & $\begin{array}{l}\text { Not } \\
\text { visible }\end{array}$ & Critical \\
\hline 12 & $\begin{array}{l}\text { Unilorin } \\
\text { Road }\end{array}$ & Right & $8^{\circ} 28^{\prime} 08.02^{\prime \prime} \mathrm{N}$ & 4³9'10.01"E & Steel & Triangle & $\begin{array}{l}\text { Informati } \\
\text { on }\end{array}$ & 2.1 & $\begin{array}{l}\text { Not } \\
\text { visible }\end{array}$ & Critical \\
\hline 13 & $\begin{array}{l}\text { Unilorin } \\
\text { Road }\end{array}$ & Left & $8^{\circ} 28^{\prime} 06.17^{\prime \prime} \mathrm{N}$ & $4^{\circ} 39^{\prime} 13.63 " \mathrm{E}$ & Steel & Round & $\begin{array}{l}\text { Informati } \\
\text { on }\end{array}$ & 2.1 & $\begin{array}{l}\text { Not } \\
\text { visible }\end{array}$ & Critical \\
\hline 14 & $\begin{array}{l}\text { Unilorin } \\
\text { Road }\end{array}$ & Left & $8^{\circ} 28^{\prime} 01.60^{\prime \prime} \mathrm{N}$ & $4^{\circ} 39^{\prime} 21.70 " \mathrm{E}$ & Steel & Round & $\begin{array}{l}\text { Informati } \\
\text { on }\end{array}$ & 2.1 & $\begin{array}{l}\text { Not } \\
\text { visible }\end{array}$ & Critical \\
\hline 15 & $\begin{array}{l}\text { Unilorin } \\
\text { Road }\end{array}$ & Left & $8^{\circ} 28^{\prime} 02.55^{\prime \prime} \mathrm{N}$ & $4^{\circ} 39^{\prime} 43.53^{\prime \prime E}$ & Steel & Rectangle & Warning & 2.1 & NIL & Good \\
\hline 16 & $\begin{array}{l}\text { Unilorin } \\
\text { Road }\end{array}$ & Left & $8^{\circ} 28^{\prime} 07.00^{\prime \prime N}$ & $4^{\circ} 39^{\prime} 47.71 " \mathrm{E}$ & Steel & Square & Warning & 2.1 & $\begin{array}{l}\text { Low } \\
\text { visibility }\end{array}$ & Fair \\
\hline 17 & $\begin{array}{l}\text { Unilorin } \\
\text { Road }\end{array}$ & Right & $8^{\circ} 28^{\prime} 25.48^{\prime \prime} \mathrm{N}$ & $4^{\circ} 40^{\prime} 10.25^{\prime \prime E}$ & Steel & Round & Warning & 2.1 & $\begin{array}{l}\text { Not } \\
\text { visible }\end{array}$ & Critical \\
\hline 18 & $\begin{array}{l}\text { Unilorin } \\
\text { Road }\end{array}$ & Right & $8^{\circ} 28^{\prime} 23.22^{\prime \prime} \mathrm{N}$ & $4^{\circ} 40^{\prime} 07.10^{\prime \prime} \mathrm{E}$ & Steel & Round & Warning & 2.1 & $\begin{array}{l}\text { Not } \\
\text { visible }\end{array}$ & Critical \\
\hline 19 & $\begin{array}{l}\text { Unilorin } \\
\text { Road }\end{array}$ & Left & $8^{\circ} 28^{\prime} 24.73^{\prime \prime} \mathrm{N}$ & $4^{\circ} 40^{\prime} 08.48^{\prime \prime} \mathrm{E}$ & Steel & Triangle & $\begin{array}{l}\text { Informati } \\
\text { on }\end{array}$ & 2.1 & $\begin{array}{l}\text { Not } \\
\text { visible }\end{array}$ & Critical \\
\hline 20 & $\begin{array}{l}\text { Unilorin } \\
\text { Road }\end{array}$ & Right & $8^{\circ} 28^{\prime} 36.42^{\prime \prime N}$ & $4^{\circ} 40^{\prime} 20.09 " \mathrm{E}$ & Steel & Round & Warning & 2.1 & $\begin{array}{l}\text { Not } \\
\text { visible }\end{array}$ & Critical \\
\hline
\end{tabular}

\subsection{Sign Condition}

While some of the signs are rated "Good', some others are rated 'Fair' or 'Critical'. Figures 1 and 2 are samples of the traffic signs rated 'Good' and are located at Chainage (Ch) $0+145 \mathrm{~m}$ and $\mathrm{Ch} 0+175 \mathrm{~m}$ on Unity Road respectively; their letterings and signs symbols are legible and the colors considered as bright. 


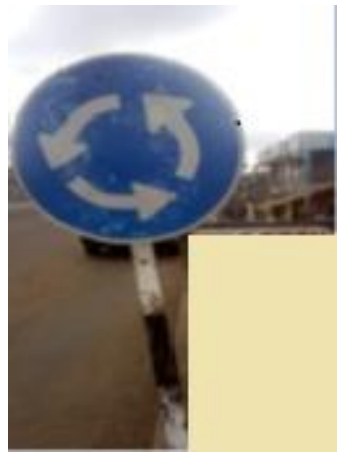

Figure 1 Signage at $0+145 \mathrm{~m}$ on Unity Rd

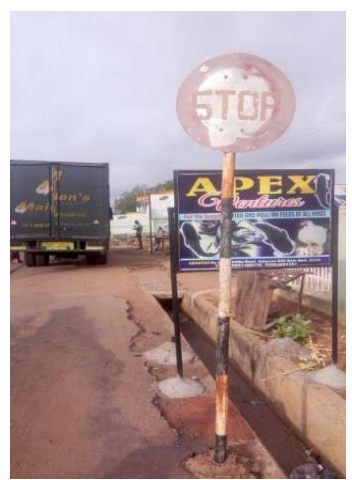

Figure 3 Signage at 0+075m on Murtala Moh'd Rd

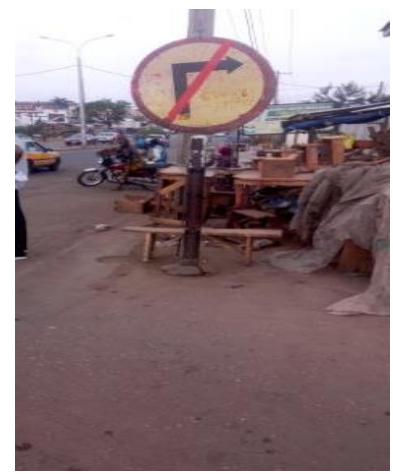

Figure 2 Signage at $0+175 \mathrm{~m}$ on Unity Rd

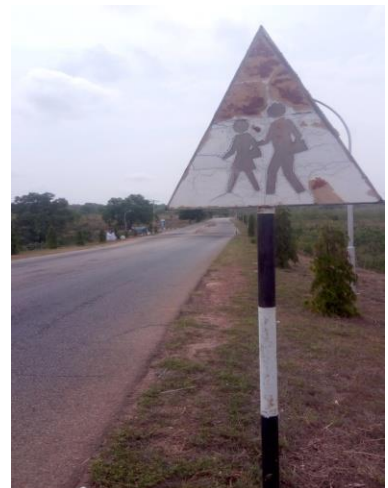

Figure 4 Signage at $2+081 \mathrm{~m}$ on University $\mathrm{Rd}$

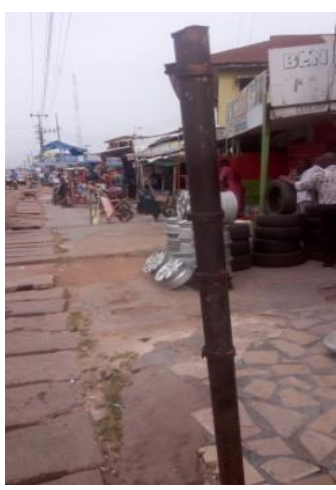

Figure 5 Signage at $0+052 \mathrm{~m}$ on Ibrahim Taiwo Rd

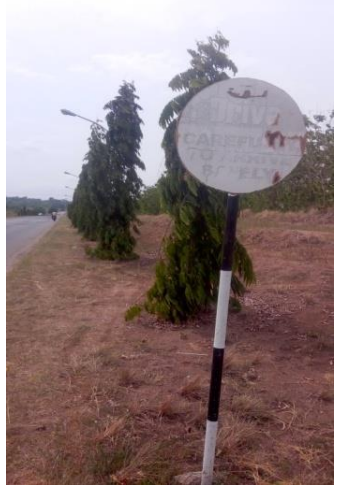

Figure 6 Signage at $2+252 \mathrm{~m}$ on University Rd

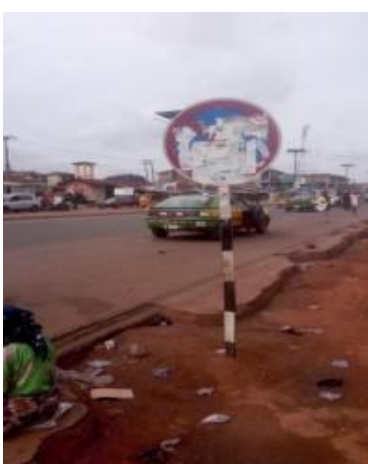

Figure 7 Signage at $1+038 \mathrm{~m}$ on Murtala Mohammed Rd
Signs on Figures 3 and 4 are classified as 'Fair' (maintenance need is futuristic) needing maintenance in the future. Their colors and legibility are already poor and need to be attended to in the very near future. Figure 5 is a signage at $\mathrm{Ch} 0+052 \mathrm{~m}$ on Ibrahim Taiwo Road. The sign mounted on the post has been removed and rated as a 'Critical' traffic sign as it is blunt and does not convey any message; therefore needs immediate maintenance. So also is the sign on Figure 6 at $\mathrm{Ch} 2+252 \mathrm{~m}$ on University of llorin roadway rated 'Critical' sign. Figure 7 is a defaced signage at Ch $1+038 \mathrm{~m}$ on Murtala Mohammed Road, its condition is classified as critical and needs urgent maintenance.

\subsection{Comparison of the Sign Post Factors}

Figures $8-12$ display a comparison in percentages of the different sign post factors between the four studied roads. Figure 4 shows that $81.8 \%$ of the signages along Unity road have no defects while all the signages along Taiwo road have one defect or the other. Forty percent of the sign posts along Taiwo road have their mounted heads removed making it impossible to identify what type of information they are to convey. In Figure 12 all the signages along Taiwo road are 'Critical' whereas $81.8 \%$ of the signages along Unity road are rated 'Good'. 
3.3 Database Management

Figure 13 is a display of the traffic signs at Murtala Mohammed Road while Figure 14 shows the signs situated along University of Ilorin Main Campus Road.

The red points represent the 'Critical' signs, the green points represent the 'Good' signs and the purple points represent the 'Fair' traffic signs. The graphical display of the sign locations assists in the maintenance gang deployment and maintenance schedule. Figures 15 and 16 respectively show the outcome of the spatial search query for the attribute tables of 'Critical' traffic signs on the University Road and the 'Good' traffic signs on the same road.

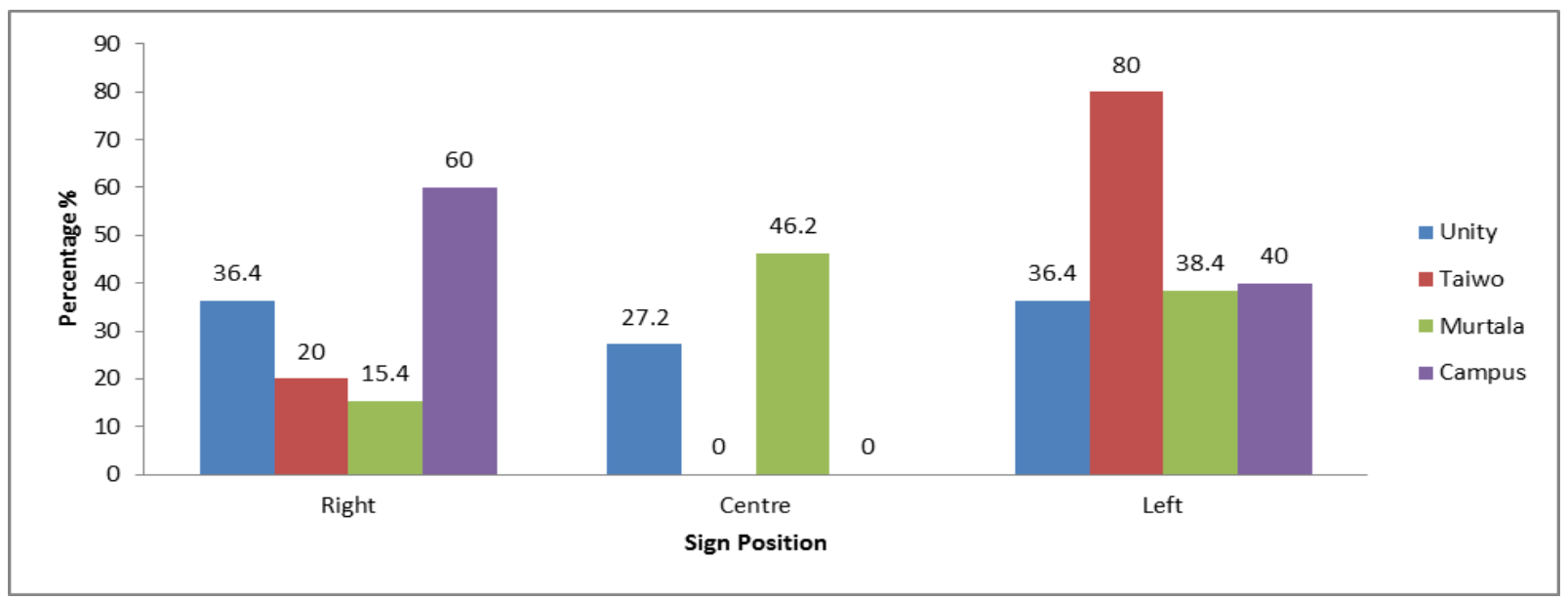

Figure 8 Comparison of the Sign Position

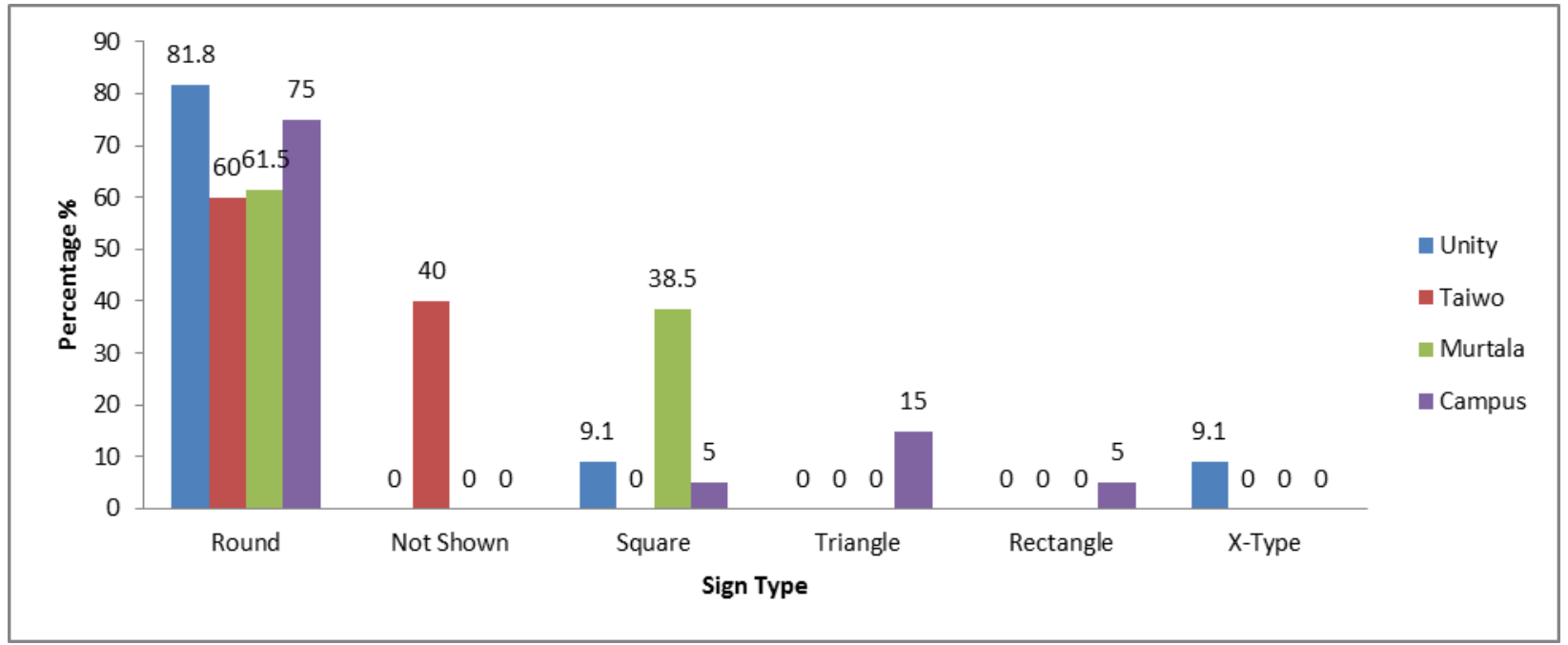

Figure 9 Comparison of the Sign Type 


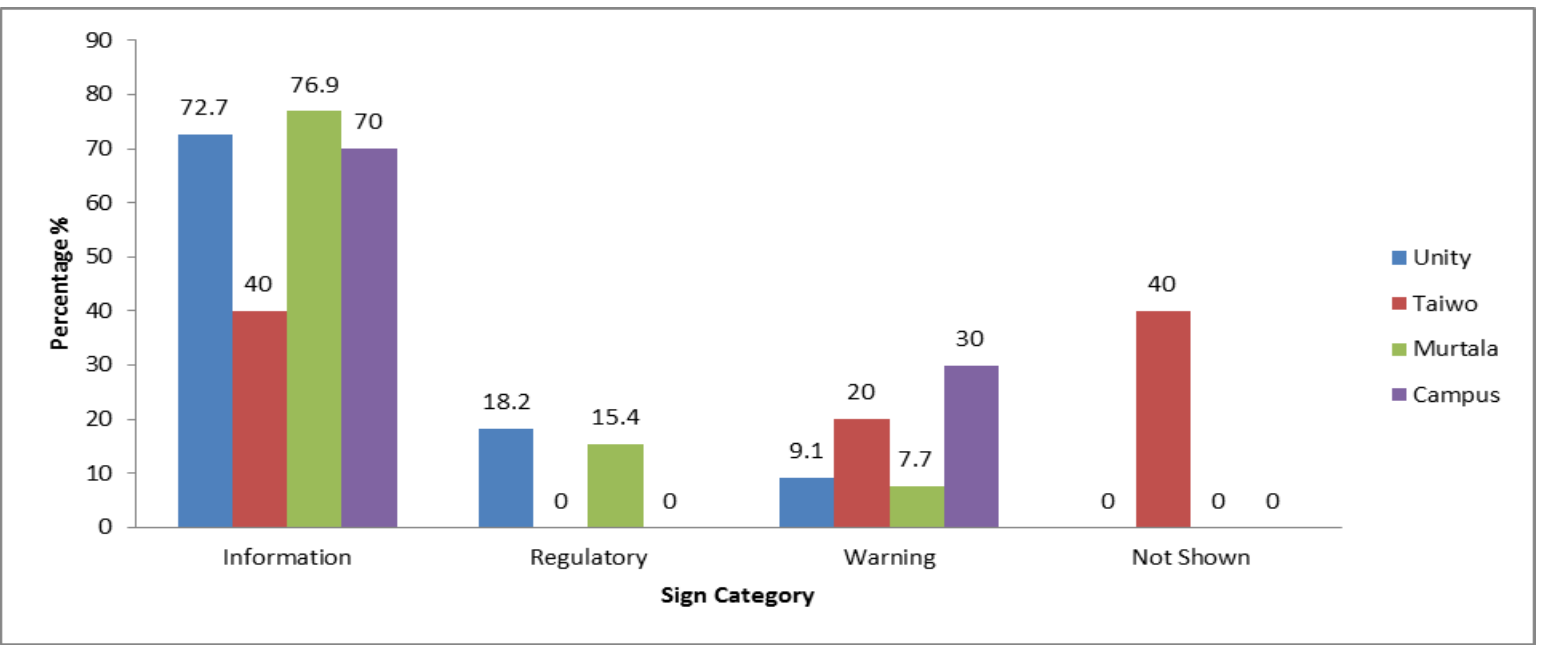

Figure 10 Comparison of the Sign Category

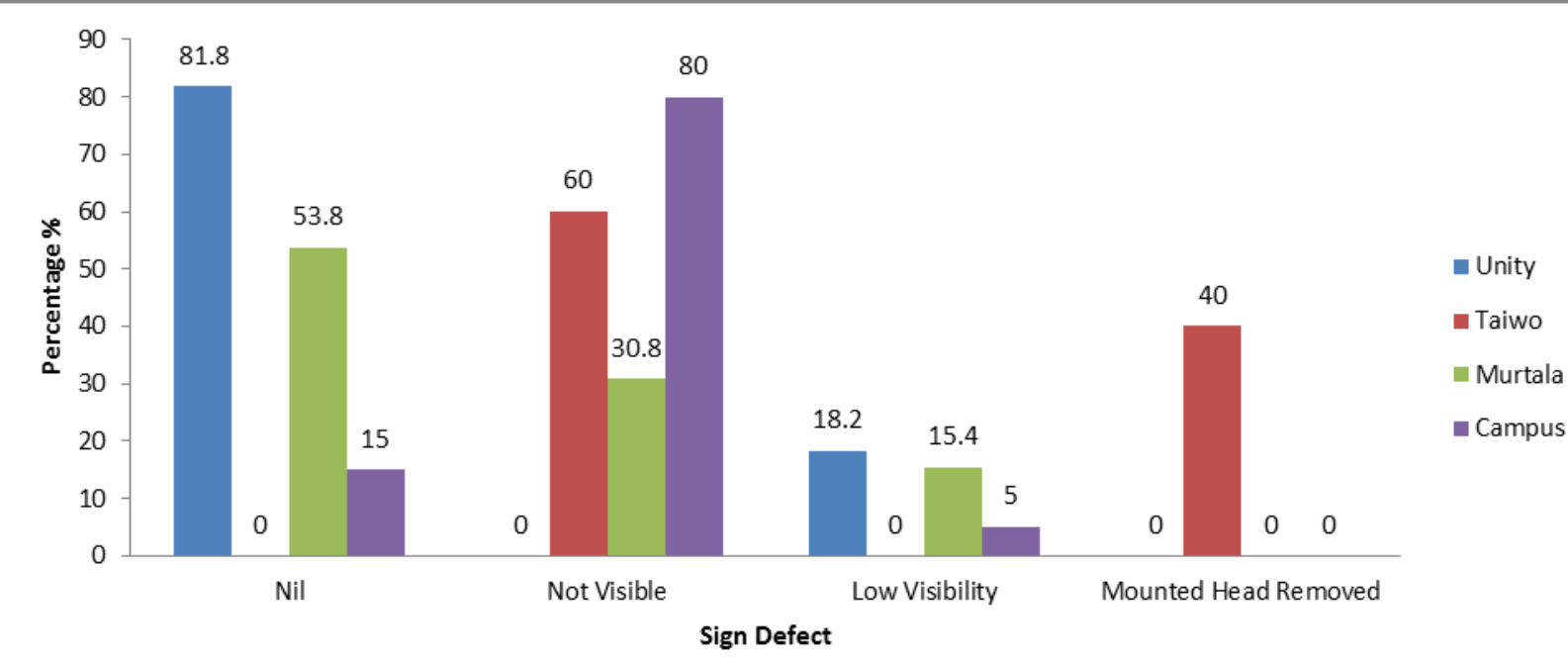

Figure 11 Comparison of the Sign Defect

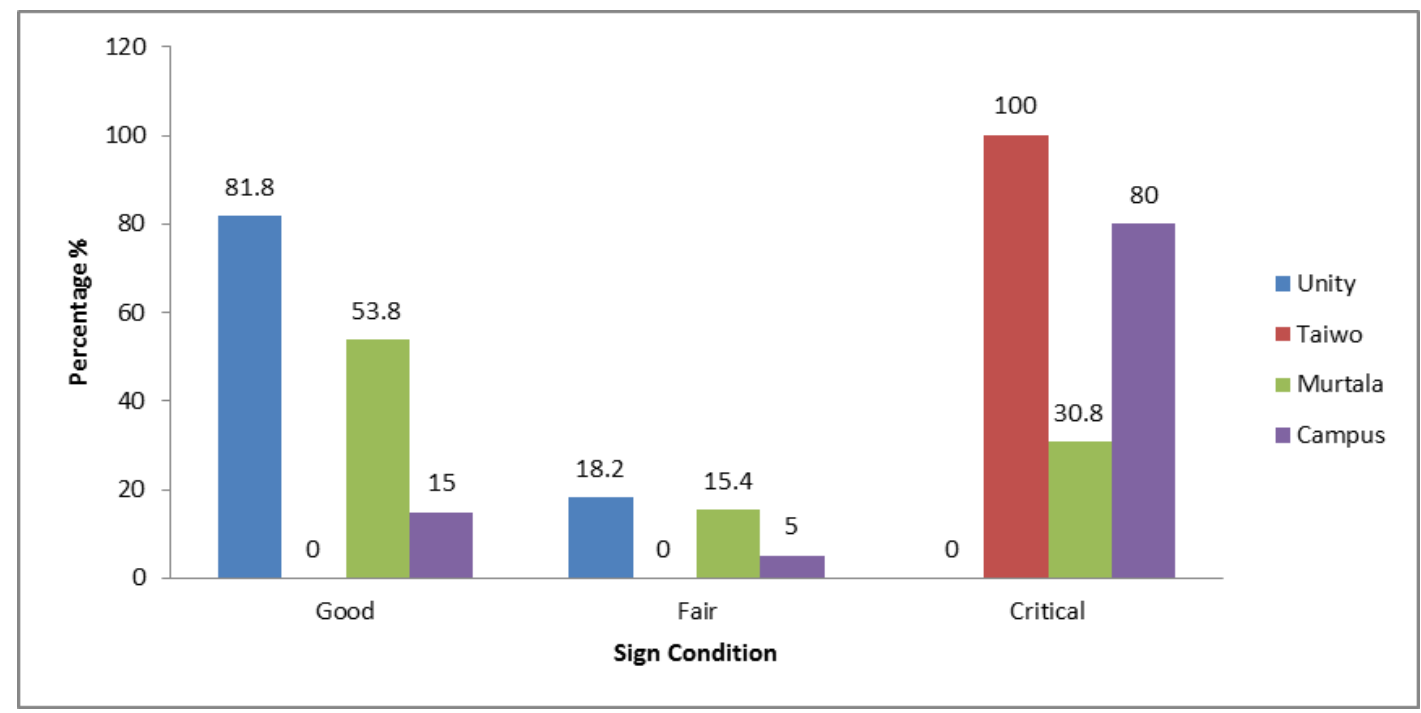

Figure 12 Comparison of the Sign Condition 


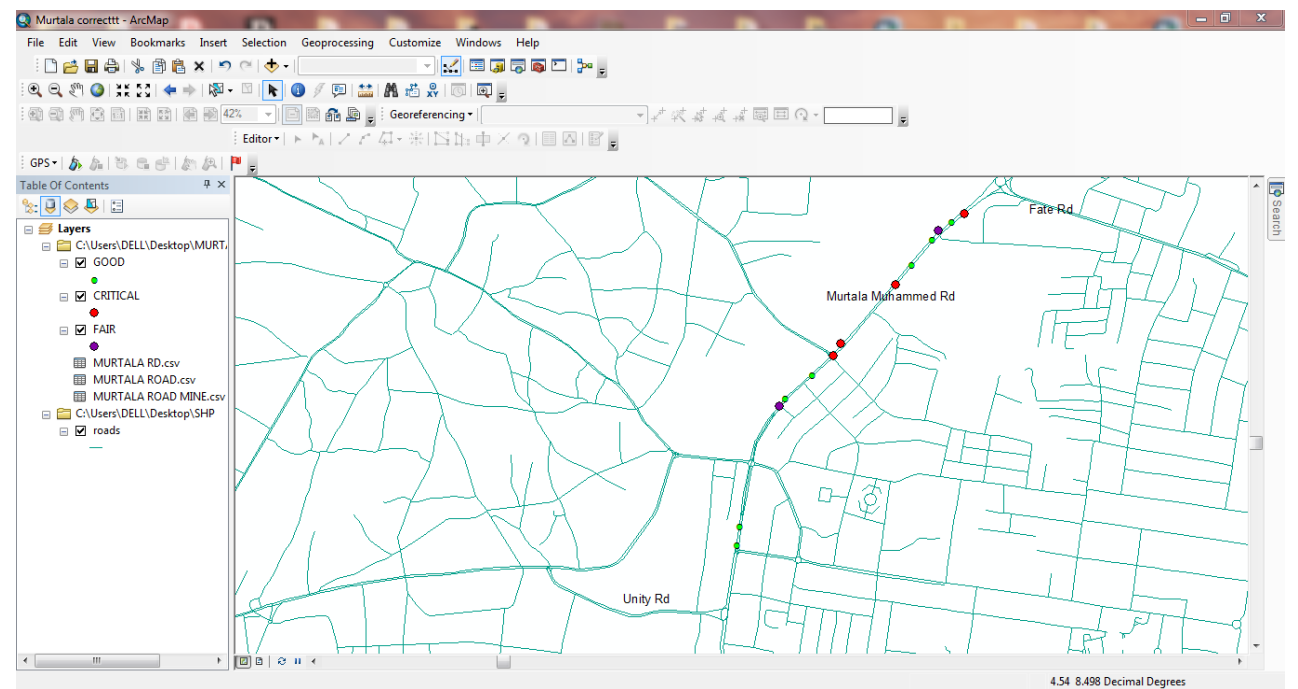

Figure 13 Traffic Signs Location at Murtala Mohammed Road

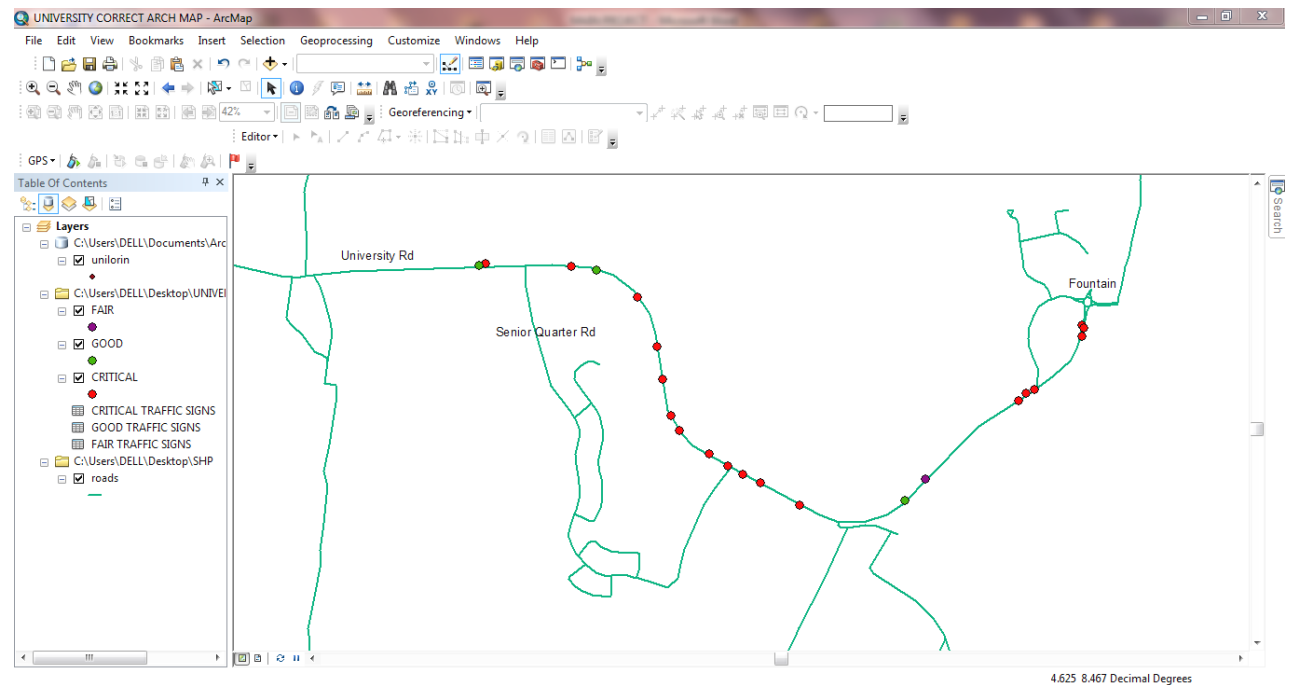

Figure 14 Traffic Signs Location at University of Ilorin Main Campus Road

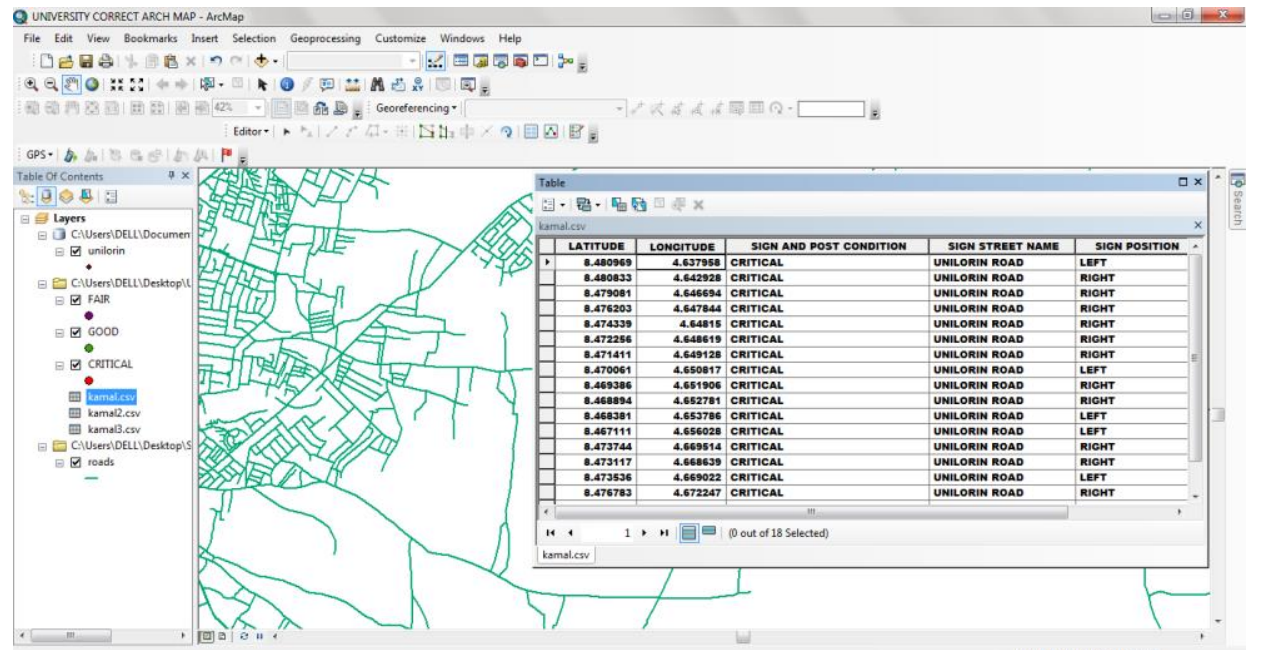

Figure 15 Attribute of collected data for Critical Traffic Signs at University of Ilorin Road 


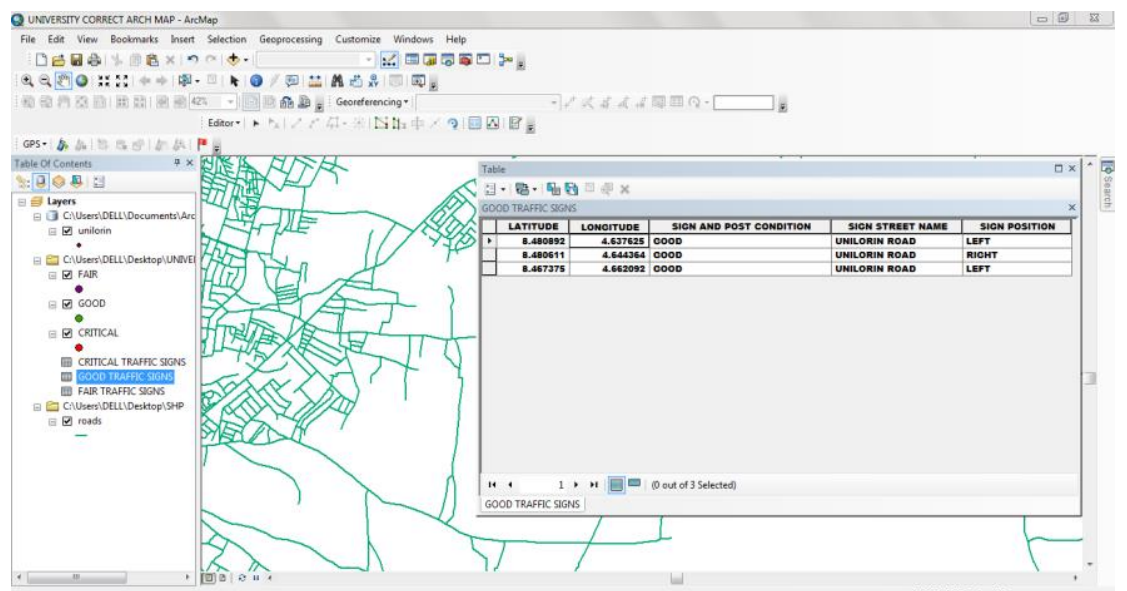

Figure 16 Attribute of collected data for Good Traffic Signs at University of Ilorin Road

Spatial search was used to query road traffic signs based on their location and / or attributes from the database. The process used to carry out the query operation involved opening of the attribute table on the layer menu. Then clicking on "select by attributes" on the table menu. In doing this, it is easy to query the database to display the critical, good and the fair traffic signs, their locations, the sign materials and other information, for easy access to the traffic management/maintenance personnel. Figure 17 for example displays the outcome of the search 'SIGN_ POST CONDITION=GOOD AND SIGN_POSITION=CENTRE' for Unity Road while Figure 18 is the outcome of the search 'SIGN_POST CONDITION=CRITICAL' for University of Ilorin Main Campus Road.

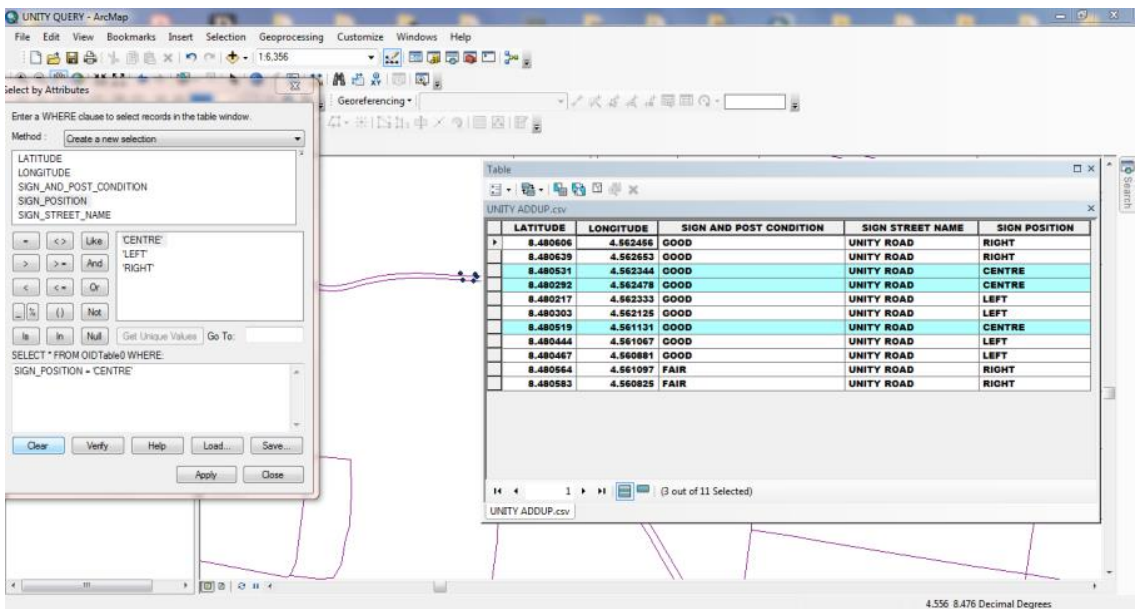

Figure 17 Result of query by Attributes showing all the good traffic signs at Unity Road

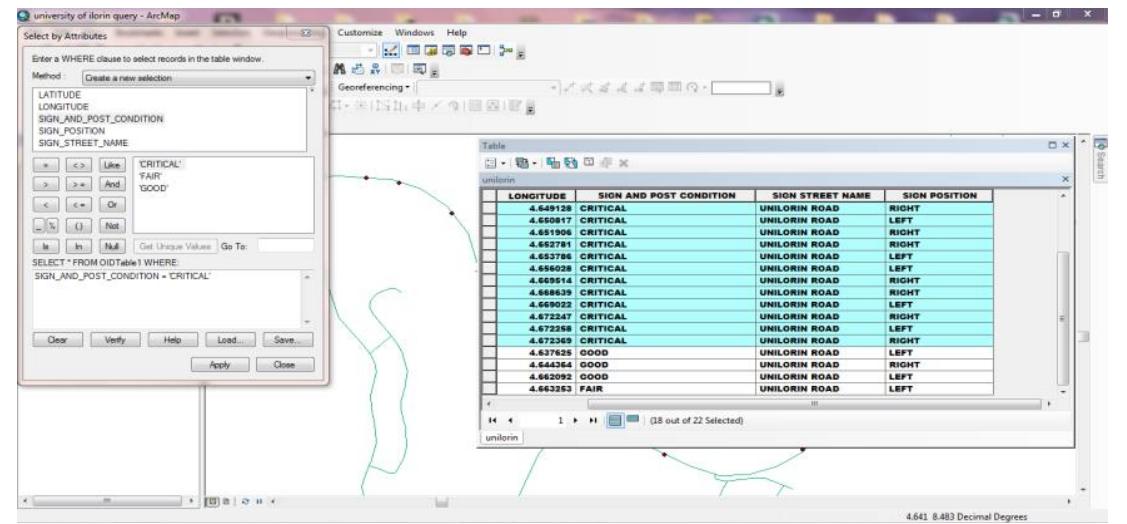

Figure 18 Result of query by Attributes showing all the critical signs on University of Ilorin Main Campus Road 


\subsection{CONCLUSION}

\subsection{Conclusion}

The study designed and developed a GPS-GIS-based traffic sign asset management system. The study identified the critical, good and the fair traffic road signs along the Unity road, University of Ilorin Main Campus road, Murtala Mohammed road and Ibrahim Taiwo road in Ilorin Metropolis, Nigeria. The study showed that Unity road has nine good traffic signs and two fair traffic signs. At Ibrahim Taiwo road, the total number of traffic signs available is five and all are rated as critical traffic signs. At Murtala Mohammed road the total number of critical traffic signs is four, the good traffic signs is seven while the fair traffic signs is two. At University of llorin road, the number of critical traffic signs is sixteen and the number of good traffic signs is three while the number of fair traffic signs is one. The ArcGIS management system successfully captured the geographic locations of all the traffic signs and their attributes thereby enhancing visual display of the results of database queries on the condition of the traffic signs on each of the studied arterial. The study further identified locations where traffic signs should be placed but are lacking. The designed and developed database can be easily updated.

\subsection{Recommendation}

It is recommended that traffic road sign management agencies should adopt the use of the developed GPS-GIS based Traffic Sign Asset Management System for the management of traffic signs, pavement markings and road safety assets rather than manual method. Future study will explore the utilization of the advantages inherent in Laser Scanner technology for the development of a Laser Scanner- GIS- based road signage management system. In the proposed study the scans of the signages and asset data will be combined in GIS to build the system.

\section{References}

[1] Alberta Infrastructure and Transportation. 2006. Alberta Highway Guide and Information Sign Manual, Alberta Infrastructure and Transportation. www.transportation.alberta.ca. Accessed $25^{\text {th }}$ January, 2020.

[2] Bhawani, C. 2014. What is GPS - How does it work, and what are its types? https://phoneradar.com/gps-global-positioning-system/. Accessed 27th March, 2020.

[3] Boggs, W., K. Heaslip and C. Louisell. 2013. Analysis of Sign Damage and Failure: Uttah Case Study. Transportation Research Record. Transportation Research Board of the National Academies, Washington DC. www.jounals.sagepub.com. Accessed 24th January, 2020.
[4] Charlton, S. G. 2006. Conspicuity, Memorability, Comprehension, and Priming in Road Hazard Warning Signs. Accident Analysis and Prevention 38(3): 496-506.

[5] Goodchild, M.F., and K.K. Kemp. 1990. NCGIA Core Curriculum in GIS https://geogra.uah.es/patxi/gisweb/GISModule/GISTheory.htm. Accessed $27^{\text {th }}$ March, 2020.

[6] Federal Highway Administration. 2009. Manual on Uniform Traffic Control Devices. US Department of Transportation, Federal Highway Administration, USA.

[7] Federal Ministry of Works. 2013. Highway Manual Part 1: Design Vol. VI, Road Traffic Signs and Road Markings. Federal Ministry of Works, Nigeria.

[8] Escobar, F., G. Hunter, I. Bishop and A. Zeger. 2020. Introduction to GIS. Department of Geomatics, The University of Melbourne http://www.sli.unimelb.edu.au/gisweb/. Accessed 27th March, 2020.

[9] Government of Alberta. 2005. Placement of Signs. http://www.transportation.alberta.ca/Content/docType233/Producti on/29SignPlacement-General.pdf. Accessed 3rd February, 2019.

[10] Punch 2019. https://punchng.com/firm-unveils-made-in-nigeriaroad-marking-paint. Accessed 3rd January 2019.

[11] Kiema, J. B. K and J. M. Mwangi. 2009. A Prototype GIS-based Road Pavement Information and Management System. Journal of Civil Engineering Research and Practice, 6(1): 79-90

[12] Ozelim L and R. E. Turochy. 2014. Modeling Retro Reflectivity Performance of Thermoplastic Pavement Markings in Alabama. Journal of Transportation Engineering 140(6): 1-6

[13] Migletz, J., J. K. Fish and J. L. Graham. 1994. Roadway Delineation Practices Handbook. US Dept. of Transport, Federal Highway Administration, USA.

[14] McGee, H. W. 2010. Maintenance of Signs and Sign Supports: A Guide for Local Highway and Street Maintenance Personnel. US Department of Transportation, Federal Highway Administration, Office of Safety.

[15] Ng, A. W., and A. H. Chan. 2008. The Effects of Driver Factors and Sign Design Features on the Comprehensibility of Traffic Signs. Journal of Safety Research, 39(3): 321-328.

[16] Nigerian National Council on Works. 2017. Communiqué of the $23^{\text {rd }}$ Meeting of The National Council On Works, Nigeria, Held at The NAF Conference Centre, Kado District, Federal Capital Territory, Abuja, Nigeria, Federal Ministry of Works, Nigeria.

[17] Ogden, M. A., K. N. Womack and J. M. Mounce. 1990. Motorist Comprehension of Signing Applied in Urban Arterial Work Zones. Transportation Research Record, 1281: 127-135.

[18] Pandey, J. and D. P. Joshi. 2011. GIS Based Road Information and Management System: A Case Study of 2010 Disaster in Uttarakhand. Journal of Engineering Research and Studies, 2(3): 124 - 127

[19] Praprut, S and P. M. Adam. 2011. Guidance for Cost-Effective Selection of Pavement Marking Materials for Work Zones. Journal of Infrastructure Systems, 17: 55-65.

[20] Pour-Rouholamin, M., and H. Zhou. 2016. Investigating the Risk Factors Associated with Pedestrian Injury Severity in Illinois. Journal of Safety Research, 57: 9-17.

[21] Rahman, S. A. U. and A. K. Duggal. 2015. Suitability of Different Materials Used for Road Marking: A Review. International Research Journal of Engineering and Technology (IRJET), 02(02): 622-625.

[22] Texas Department of Transportation. 2004. Pavement Marking Handbook. Texas Department of Transportation, USA.

[23] United Nations. 1968. Vienna Convention on Road Signs and Signals. United Nations. Stopline January, 2020.

[24] Yannis, G., A. Kondyli and X. Georgopoulou. 2014. Investigation of the Impact of Low Cost Traffic Engineering Measures on Road Safety in Urban Areas. International Journal of Injury Control and Safety Promotion, 21(2): 181-189. 